\title{
Artificially Intelligent Technology for the Margins: A Multidisciplinary Design Agenda
}

\author{
Franziska Tachtler \\ TU Wien \\ Vienna, Austria \\ franziska.tachtler@tuwien.ac.at \\ Mohammed Khwaja \\ Imperial College London \\ London, UK \\ mohammed.khwaja16@imperial.ac.uk \\ Shaimaa Lazem \\ City of Scientific Research and \\ Technological Applications \\ Alexandria, Egypt \\ slazem@srtacity.sci.eg
}

\author{
Konstantin Aal \\ Tanja Ertl \\ konstantin.aal@uni-siegen.de \\ tanja.ertl@uni-siegen.de \\ University of Siegen \\ Germany
}

Reem Talhouk

Northumbria University

Newcastle, UK

reem.talhouk@northumbria.ac.uk

\author{
Aneesha Singh \\ University College London \\ London, UK \\ aneesha.singh@ucl.ac.uk
}

\author{
Daniel Diethei \\ Jasmin Niess \\ diethei@uni-bremen.de \\ niessj@uni-bremen.de \\ University of Bremen \\ Germany
}

\author{
Giovanna Nunes Vilaza \\ Technical University of Denmark \\ Copenhagen, Denmark \\ gnvi@dtu.dk
}

Marguerite Barry

University College Dublin

Dublin, Ireland

marguerite.barry@ucd.ie

\author{
Volker Wulf \\ University of Siegen \\ Siegen, Germany \\ volker.wulf@uni-siegen.de
}

\begin{abstract}
There has been increasing interest in socially just use of Artificial Intelligence (AI) and Machine Learning (ML) in the development of technology that may be extended to marginalized people. However, the exploration of such technologies entails the development of an understanding of how they may increase and/or counter marginalization. The use of AI/ML algorithms can lead to several challenges, such as privacy and security concerns, biases, unfairness, and lack of cultural awareness, which especially affect marginalized people. This workshop will provide a forum to share experiences and challenges of developing AI/ML health and social wellbeing technologies with/for marginalized people and will work towards developing design methods to engage in the re-envisioning of $\mathrm{AI} / \mathrm{ML}$ technologies for and with marginalized people. In doing so we will create cross-research area dialogues and collaborations. These discussions build a basis to (1) explore potential tools to support designing $\mathrm{AI} / \mathrm{ML}$ systems with marginalized people, and (2) develop a design agenda for future research and AI/ML technology for and with marginalized people.
\end{abstract}

Permission to make digital or hard copies of part or all of this work for personal or classroom use is granted without fee provided that copies are not made or distributed for profit or commercial advantage and that copies bear this notice and the full citation on the first page. Copyrights for third-party components of this work must be honored.

For all other uses, contact the owner/author(s).

CHI '21 Extended Abstracts, May 8-13, 2021, Yokohama, Japan

(C) 2021 Copyright held by the owner/author(s)

ACM ISBN 978-1-4503-8095-9/21/05.

https://doi.org/10.1145/3411763.3441333

\author{
Geraldine Fitzpatrick \\ TU Wien \\ Vienna, Austria \\ geraldine.fitzpatrick@tuwien.ac.at
}

\section{CCS CONCEPTS}

- Human-centered computing $\rightarrow$ Participatory design; User centered design; - Security and privacy $\rightarrow$ Human and societal aspects of security and privacy; - Computing methodologies $\rightarrow$ Machine learning; Artificial intelligence.

\section{KEYWORDS}

AI, ML, Data, Privacy, Security, Marginalized people, Global South, HCI4D, ICT4D, Ethics

\section{ACM Reference Format:}

Franziska Tachtler, Konstantin Aal, Tanja Ertl, Daniel Diethei, Jasmin Niess, Mohammed Khwaja, Reem Talhouk, Giovanna Nunes Vilaza, Shaimaa Lazem, Aneesha Singh, Marguerite Barry, Volker Wulf, and Geraldine Fitzpatrick. 2021. Artificially Intelligent Technology for the Margins: A Multidisciplinary Design Agenda. In CHI Conference on Human Factors in Computing Systems Extended Abstracts (CHI '21 Extended Abstracts), May 8-13, 2021, Yokohama, Japan. ACM, New York, NY, USA, 7 pages. https://doi.org/10.1145/3411763.3441333

\section{BACKGROUND}

Artificial Intelligence (AI) is increasingly being promoted and developed for different areas such as the public sector, social care and public health. Indeed, such technologies are being applied and/or proposed as tools for addressing multiple factors that constitute the social determinants of health and not just clinical healthcare services. Especially in the context of marginalized communities, 
the capability to navigate social and welfare services, such as housing, educational and rehabilitation services, are as important to promoting health and social wellbeing as navigating healthcare services [42, 43]. Researchers, management and strategy consulting companies argue for the potential of integrating AI technologies for the social good as they are projected to improve decision making and the effective management of public- and social-sector entities [9], catch fraud in the insurance system [32], enable automated diagnostic systems in remote areas [13] or help allocating homeless youth to housing services [4]. Furthermore, De-Artega et al. [12] introduced the term machine learning for the developing world (ML4D), where they highlight the potential for ML technologies, including applications and data, in addressing the societal challenges in developing countries. The authors present a road map for future work on ML4D, suggesting to improve data reliability, provide direct solutions and inform policy and decision makers.

However, despite the increasing interest in the design, development and deployment of AI/ML technologies, gaps still remain regarding its adoption by marginalized groups (e.g., the 'Global South', people of lower socio-economic status, refugees, asylum seekers, homeless people, prisoners and people with multiple disabilities). Recent works highlight that challenges around technology design for marginalized people have persisted and are more pressing than ever [40]. For instance, not taking into the account the specific needs of marginalized communities in the design of AI/ML health technologies lead to the widening of treatment gaps [31], and potential harms concerning data-injustice $[11,15]$. There is a clear need for further efforts to design with and for the margins, especially when it comes to using artificially intelligent approaches [25]. The use of AI in the public social, welfare and health services leads to a vicious cycle of AI-based discrimination that may further marginalize people [8].

Therefore, we propose to use this one-day workshop as a space for HCI and AI researchers, designers and third and public sector support organisations to come together and discuss cases, experiences and challenges that relate to AI/ML technologies for and with marginalized people. In particular, we want to focus on exploring and developing novel design methods and approaches to engage with marginalized people in the design of $\mathrm{AI} / \mathrm{ML}$ technologies as well as providing a set of overarching practice principles to guide our community's work.

This workshop is motivated by two main research gaps identified in the literature. First, knowledge and data obtained from studies with 'mainstream' groups cannot be directly applied to minorities. There is, therefore, a need to work with marginalized people to overcome challenges in obtaining data, related to lack of trust and transparency, to build more inclusive data sets and algorithms. Second, as a starting point to tackle this issue, there should be more efforts on the development of design tools to engage the wide variety of different marginalized people and take their diverse needs into account. We expand on these points in the following sections.

\subsection{Moving from the Mainstream to the Margins}

A successful deployment of AI/ML technologies requires the creation of relevant data sets based on data shared by end-user groups.
Ownership, data control and knowledge are also key elements in existing data-justice principles [22]. However, in a recent systematic review of HCI literature on ML systems for mental health technologies, the authors emphasize that challenges in the development of ML solutions have even bigger consequences for marginalized people. First, the accuracy of AI/ML solutions depends on where and with which populations the algorithms are developed and trained, which means that models created for one context cannot be transferred and deployed in another [40]. Such challenges may be extended to other health related domains and to those within social care and the public sector (e.g., housing). Given that mainstream efforts within AI applications are constrained to a select set of nations and groups, the results may further reinforce discrimination against minorities [46]. In addition, demographic disparities may be further magnified in the context of sensitive domains where stigma already hinders engagement in data collection processes $[16,36]$. Therefore, it is an open challenge about how future initiatives should strive for more diversity and inclusion in order to mitigate the risk of bias.

Furthermore, designing for trust among marginalized people is essential, as shown by previous studies with, refugees, asylum seekers [16, 36], homeless youth [1], and prisoners [17]. One of our goals in this workshop is to develop a deeper understanding of whether and how vulnerable populations develop trust in AIdriven services and to work on a more differentiated picture of their trusting stance towards such technology. For instance, in line with work from McKnight et al. [29] on trust in technology, previous research on marginalized people suggests that one has to differentiate between the lack of initial trust as a barrier to starting engaging with AI-based technology (e.g., sharing data) and the lack of knowledge-based trust [29], which builds on previous trustortrustee interactions (e.g., distrust while already using AI-based technology). In addition to the different types of trust, contextual factors also seem to be an essential factor when building trust. A recent study suggests that public trust in $\mathrm{AI}$ is lower for sensitive contexts [3].

To illustrate, marginalized people such as refugees and asylum seekers have been shown to have high privacy and security concerns [24] coupled with low agency in detailing what data they are willing to share [10,33]. In addition, stigma and vulnerability are some of the harmful consequences of data leakage, which may have a stronger impact within already marginalized people [28]. The lack of trust and fear of being treated negatively additionally hinders accessing services and causes further marginalization [27, 36] but is also a barrier to accessing technological driven services. Previous research shows that transparency and choice over how the data collected is used (and re-used) have an important role in the perception of trustworthiness of the technologies using/asking to share data, but existing initiatives are failing to provide that [44]. Thus, we need better tools and methods to support designing more trustworthy technologies which provide reassurance to marginalized people who had negative experiences with different services and fear discrimination. 


\subsection{Striving to Design for Inclusion and Diversity}

The aforementioned challenges are underpinned by the lack of meaningful and active inclusion of marginalized people in the design of AI/ML technologies. However, designing with marginalized people presents its own challenges [7, 19, 35, 45]. It has been identified that even in non-marginalized contexts there is a lack of designing human-friendly which involve target-populations [40, 47]. In previous $\mathrm{CHI}$ workshops it has been emphasized that $\mathrm{HCI}$ researchers need more accessible, intuitive and easier-to-use tools to facilitate an inclusive design process [21]. Despite recent efforts exploring how to create design tools (e.g., for explainable AI systems [26]), such works have not been focused on engaging with marginalized people. Consequently, we are aiming to provide a platform to discuss possible starting points on how to engage marginalized people in the design process.

Researchers working with people with disabilities argue for including people with disabilities in the development of models of AI systems and point out inclusive design, participatory design and value based design as promising approaches [41]. In addition, successful intercultural communication within the design process can promote its implication in the technical development, which in turn helps building trust through the use of the application itself [16]. Indeed, communicating the nature of the technology through participatory or community-based design processes [20,48-50] may not only increase the understanding among marginalized groups but will also enable the design of $\mathrm{AI} / \mathrm{ML}$ technologies based on their principles and understanding of data-justice, trust and transparency.

However, different marginalized groups have different needs [18, 40]. For instance, prisoners' opportunities for technical participation in the design process are massively restricted as they are not trusted to use technologies without criminal intention. Additionally, post-colonial $\mathrm{HCI}$ has identified several intercultural challenges of designing technologies in the 'Global South' $[6,23]$. Overall, the aforementioned literature highlights the need to develop a better understanding of how to design AI/ML technologies with and for specific marginalized populations in order to counter challenges related to transferability and data-justice.

\subsection{Workshop Goals}

Designing inclusive $\mathrm{AI} / \mathrm{ML}$ technologies is a field that involves stakeholders from multiple areas with a variety of different backgrounds. Consequently, we aim to utilise virtual platforms to broaden participation in our one-day workshop. We are striving to include participants with a variety of different backgrounds such as $\mathrm{HCI}$ scholars, AI/ML researchers, non-academic community organisations and people from marginalized communities - that either have experience/interest in $\mathrm{AI} / \mathrm{ML}$ technologies, and/or in working with marginalized people.

The workshop will promote knowledge and experience exchange particularly in relation to the previously outlined challenges. Through the workshop we aim to formulate novel approaches/methods and principles for designing $\mathrm{AI} / \mathrm{ML}$ technologies for and with marginalized people.

The key objectives of this workshop are:
(1) bringing together communities working with marginalized people and exchanging experiences on working with developing and investigating data driven technologies

(2) identifying and developing a design agenda for how to design/develop AI/ML technologies with/for marginalized people and for the design itself of the AI/ML driven technologies

(3) ideating processes and tools for designing/ developing AI/ML technologies with/for marginalized people.

These key objectives lead to the following workshop outputs:

(1) the creation of a community of cross-disciplinary researchers/ practitioners working with $\mathrm{AI} / \mathrm{ML}$ technologies and/or marginalized people

(2) a collection of guiding principles supporting designing/developing AI/ML technologies with/for marginalized people in the future

(3) a collection of initial tools/methods supporting the application of these principles.

\section{ORGANIZERS}

This group of workshop organizers reflect expertise in the intersection of working with marginalized people in multiple geographical contexts and $\mathrm{AI} / \mathrm{ML}$ and data driven technologies. Additionally, the organizers have experience running successful workshops at $\mathrm{CHI}$ and other ACM and affiliated venues [2, 5, 14, 30, 34, 37-39].

Franziska Tachtler is a $\mathrm{PhD}$ student and research associate at HCI Group, TU Wien. Her research focuses on the role of technology in promoting resilience of young asylum-seekers and their social-ecological systems. She works with young asylum-seekers, volunteer organizations and mental health service providers using participatory engagement methods.

Konstantin Aal is a $\mathrm{PhD}$ student and a research associate at the Institute for Information Systems and New Media, University of Siegen. He is part of come_ IN, a research project which founded several computer clubs for children and their relatives including refugees. His current research focuses on technology appropriation by local communities in the Global South.

Tanja Ertl is a PhD student at the Institute for Information Systems and New Media, University of Siegen. Based on the Grounded Design approach, her research focuses on the promotion of wellbeing of marginalized groups by transforming therapeutic methods into self-help tools to bridge the gaps in state care that currently exist to support integration and resocialization processes.

Daniel Diethei is a $\mathrm{PhD}$ student and researcher at the University of Bremen, Germany. As a member of the Leibniz Science-Campus Digital Public Health he works with digital technologies in medicine and healthcare with a focus on underserved populations.

Jasmin Niess is a postdoctoral researcher at the University of Bremen, Germany. Her research focuses on the design and evaluation of technologies that improve wellbeing. Her past experiences in academia and socially-relevant work engagements make her particularly enthusiastic about conducting research that improves societal wellbeing.

Mohammed Khwaja is a research scientist and a $\mathrm{PhD}$ student at Imperial College London. He investigates how data science and psychology can be merged to personalise digital interventions. His 
previous research included the exploration of differences in machine learning prediction models across different cultures.

Reem Talhouk is a Vice Chancellor Research Fellow in the School of Design and the Centre for International Development at Northumbria University. She has over 5 years of experience in designing technologies with refugees and asylum seekers in the Middle East, Europe and Australia. Her current research focuses on developing design methods for socially just humanitarian and global development innovation.

Giovanna Nunes Vilaza is a $\mathrm{PhD}$ student and research associate at Department of Health Technology, Technical University of Denmark. Her current research aims at proposing ethical and participant-centered futures for open-access data platforms for behavioral monitoring.

Shaimaa Lazem is an Associate Research Professor at SRTACity, Egypt. Her research interests include participatory design, post-colonial computing, and decolonizing HCI. Her previous projects included designing heritage documentation applications for rural populations.

Aneesha Singh is an Assistant Professor of Human Computer Interaction at the UCL Interaction Centre. She is interested in the design, adoption and use of personal health and wellbeing technologies in everyday contexts. Her research focuses on digital health, ubiquitous computing, multisensory feedback and wearable technology, especially in sensitive and stigmatised populations.

Marguerite Barry is Lecturer and Assistant Professor at the School of Information \& Communication Studies at University College Dublin. Her research focuses on developing practical ethical frameworks to inform design of AI technologies.

Volker Wulf is a computer scientist with an interest in the area of IT system design in real-world contexts, this includes the development of innovative applications from the areas of cooperation systems, knowledge management and community support. He is head of the Institute for Information Systems and New Media at the University of Siegen.

Geraldine Fitzpatrick is Professor of Technology Design and Assessment and heads the Human Computer Interaction Group at TU Wien. She is also an ACM Distinguished Scientist, IFIP TC13 Pioneer Award recipient, and IFIP Fellow. Her research is at the intersection of social and computer sciences, with a particular interest in supporting wellbeing, community building and active engagement.

\section{PRE-WORKSHOP PLANS}

The workshop will be promoted through a dedicated website that will communicate the aims and structure of the workshop and will also be updated with position papers of workshop participants and workshop outputs (see https://techforthemargins.wordpress.com). We will spread the call for submissions through relevant academic mailing lists (e.g., displaced-hci mailing list, $\mathrm{CHI}$ Announcements, ArabHCI) and social media groups (afriCHI, HCI4d Google Group, Computing and Mental Health Linkedin group, HCI across borders Facebook group). We will also actively seek submissions from our network and contacts, with a special focus on non-academic community organisations working with and for marginalized people such as (forced) migrants, as well as social activists within marginalized. A total of 20 participants (excluding organizers) will be invited to partake in the workshop based on the position papers submitted.

\section{WORKSHOP STRUCTURE}

The workshop will facilitate interactive activities among participants, clustered based on application areas (e.g., social care) and the marginalized people they are working with, in order to achieve workshop goals.

To better facilitate remote participation living across time zones and exchange among participants, we will offer pre- and postworkshop activities, such as collecting materials in advance to provoke discussions at the workshop and final reflections after the workshop. Prior to the workshop, we will create a shared platform, e.g., a google doc sheet, where the participants can introduce themselves and discuss their backgrounds, interests and challenges to establish a first exchange.

In addition, we will use platforms such as Well-Sorted to collect and group overarching challenges and themes, and offer the possibility to meet pre-conference virtually, which takes place at a different timezone than the conference, to establish first discussions and support participants to become familiar with the overall scope and idea of the workshop. In order to create a social connection at the workshop, we will ask the participants in advance that they either keep their video on or have a profile picture of themselves.

Across all phases of the workshop, we will work with digital platforms such as Miro, Well-Sorted, and Wonder. Wonder will be used as an inclusive, immersive and interactive space for all by creating several room areas for discussion and exchange. It is a robust online tool that workshop co-organisers have trialled with participants living in contexts of intermittent and weak internet connectivity. During the workshop co-organisers will act as facilitators and support groups in different rooms in documenting the discussion and adding content to digital platforms. As a backup conference tool we will use Zoom.

The 4 hour long workshop will consist of three main activities (see workshop schedule in Table 1). We may adapt the time of the workshop based on a form to be sent to accepted participants inquiring about their time zones in order to ensure that most participants can attend. In the case of participants not able to attend all of the workshop synchronously, participants will have the opportunity to participate in pre and post workshop activities asynchronously through contributing discussion points on the digital platforms Well-Sorted and Miro. By setting up the digital infrastructure in advance and involving the accepted participants in pre-workshop activities, we will ensure that participants are familiar with the tools and know each other in advance. Therefore, the barriers to participating in the workshop via a video conference tool are much lower.

Discussions in the activities will be based on the challenges and experiences presented by participants in position papers.

\section{POST-WORKSHOP PLANS}

The post-workshop activities will focus on establishing a community that engages in the development of design methods that HCI researchers can draw on when engaging in the designing of $\mathrm{AI} / \mathrm{ML}$ 


\begin{tabular}{|l|l|}
\hline Timetable (CET) & Activities \\
\hline 13:00-13:15 & Welcome \\
\hline 13:15-14:00 & $\begin{array}{l}\text { Activity 1: Understanding and situating AI/ML technologies in contexts } \\
\text { of marginalization in each application area }\end{array}$ \\
\hline 14:00-14:15 & Break \\
\hline 14:15-15:00 & Activity 2: Formulating principles that will guide future work \\
\hline 15:00-15:15 & Presenting back \\
\hline 15:15-15:30 & Break \\
\hline 15:30-16:30 & $\begin{array}{l}\text { Activity 3: Developing methods and approaches for designing with } \\
\text { marginalized groups }\end{array}$ \\
\hline 16:30-16:45 & Presenting back \\
\hline 16:45-17:00 & Wrap up and next steps \\
\hline
\end{tabular}

Table 1: Workshop Schedule in CET. We plan to adjust the schedule to the participants' time zones.

technologies for/with marginalized populations. To support future collaborations, we will discuss a means of communication through which participants can support one another, and identify collaborations/ funding streams (e.g., slack channel, series of seminars to be conducted throughout the year, meetings for further discussion) for future research in this area. In addition, we will facilitate a post$\mathrm{CHI}$ reflection meeting to discuss the outputs of the workshop and exchange our experience of the whole conference. This will help to create a sense of research community.

We will share all the documentation of the activities and workshop outputs through the workshop websites and in the form of a public facing blog post and Interactions article. Interested participants will be invited for further discussions on working towards a special issue in ToCHI that will unpack the challenges and future of AI/ML technologies and design in contexts of marginalization. Follow-up workshops and Special Interest Groups at other ACM and different disciplinary conferences in the area of health \& social wellbeing and ML will be held to further discussions and grow the community interested in this research area.

\section{CALL FOR PARTICIPATION}

The integration of Artificial Intelligence (AI) and Machine Learning (ML) into social, health, and public sectors could bring promising benefits. However, these technologies lead to several challenges, e.g., privacy concerns, unfairness, and the increase of marginalization. Thus, it is essential that more attention is given on how we design such systems for/with marginalized people.

This workshop will provide a forum to exchange experiences on designing technologies for/with marginalized people and AI/ML driven technologies. We will explore potential tools to support designing AI/ML systems with marginalized people, and develop a design agenda. We use a broad definition of marginalized people that may include, but is not limited to, people of/with low socioeconomic status, disabilities and migration histories.

We invite researchers, practitioners and community members interested in the aforementioned topic to submit a 2-4 page position paper as PDF using ACM Master Article Template. Submissions should critically reflect on current projects and/or present challenges and approaches. In particular, we encourage submissions that reflect on:

- case studies with marginalized people and/or AI/ML,

- design methods used with marginalized people that may be transferable to the design of AI/ML technologies,

- past experiences illustrating positive or negative outcomes of $\mathrm{AI} / \mathrm{ML}$ in this context.

Submissions should be sent to techforthemargins@gmail.com by February 3. Acceptance will be based on quality, relevance, and diversity and will be communicated by February 21 . At least one author of each accepted position paper must register and attend the workshop and register for at least one day of the conference.

More details on https://techforthemargins.wordpress.com/

\section{REFERENCES}

[1] Elizabeth C Adkins, Alyson K Zalta, Randy A Boley, Angela Glover, Niranjan S Karnik, and Stephen M Schueller. 2017. Exploring the potential of technologybased mental health services for homeless youth: A qualitative study. Psychological services 14, 2 (2017), 238.

[2] Ebtisam Alabdulqader, Norah Abokhodair, and Shaimaa Lazem. 2017. Designing for the Arab World. In Proceedings of the 2017 ACM Conference Companion Publication on Designing Interactive Systems (Edinburgh, United Kingdom) (DIS '17 Companion). Association for Computing Machinery, New York, NY, USA, 348-351. https://doi.org/10.1145/3064857.3064860

[3] Naomi Aoki. 2020. An experimental study of public trust in AI chatbots in the public sector. Government Information Quarterly 37, 4 (2020), 101490.

[4] Mohammad Javad Azizi, Phebe Vayanos, Bryan Wilder, Eric Rice, and Milind Tambe. 2018. Designing fair, efficient, and interpretable policies for prioritizing homeless youth for housing resources. In International Conference on the Integration of Constraint Programming, Artificial Intelligence, and Operations Research. Springer, 35-51.

[5] Marguerite Barry, Aphra Kerr, and Oliver Smith. 2020. Ethics on the Ground: From Principles to Practice. In Proceedings of the 2020 Conference on Fairness, Accountability, and Transparency (Barcelona, Spain) (FAT $\left.T^{*} 20\right)$. Association for Computing Machinery, New York, NY, USA, 688. https://doi.org/10.1145/3351095. 3375684

[6] Nicola J Bidwell. 2016. Decolonising HCI and interaction design discourse: some considerations in planning AfriCHI. XRDS: Crossroads, The ACM Magazine for Students 22, 4 (2016), 22-27.

[7] Ana Maria Bustamante Duarte, Nina Brendel, Auriol Degbelo, and Christian Kray. 2018. Participatory Design and Participatory Research: An HCI Case Study with Young Forced Migrants. ACM Trans. Comput.-Hum. Interact. 25, 1, Article 3 (Feb. 2018), 39 pages. https://doi.org/10.1145/3145472 
[8] Jan C. Weyerer and Paul F. Langer. 2019. Garbage in, garbage out: The vicious cycle of ai-based discrimination in the public sector. In Proceedings of the 20th Annual International Conference on Digital Government Research. 509-511.

[9] Michael Chui, Martin Harrysson, James Manyika, Roger Roberts, Rita Chung, Pieter Nel, and Ashley van Heteren. 2018. Applying artificial intelligence for social good. Retrieved Nov 23, 2020 from https://www.mckinsey.com/featuredinsights/artificial-intelligence/applying-artificial-intelligence-for-social-good

[10] Lizzie Coles-Kemp and Rikke Bjerg Jensen. 2019. Accessing a New Land: Designing for a Social Conceptualisation of Access. In Proceedings of the 2019 CHI Conference on Human Factors in Computing Systems (Glasgow, Scotland Uk) (CHI '19). Association for Computing Machinery, New York, NY, USA, 1-12. https://doi.org/10.1145/3290605.3300411

[11] Sasha Costanza-Chock. 2020. Design justice: Community-led practices to build the worlds we need. MIT Press.

[12] Maria De-Arteaga, William Herlands, Daniel B. Neill, and Artur Dubrawski. 2018 Machine Learning for the Developing World. ACM Trans. Manage. Inf. Syst. 9, 2, Article 9 (Aug. 2018), 14 pages. https://doi.org/10.1145/3210548

[13] Daniel Diethei and Johannes Schöning. 2018. Using smartphones to take eye images for disease diagnosis in developing countries. In Proceedings of the Second African Conference for Human Computer Interaction: Thriving Communities. 1-3.

[14] Daniel Diethei, Julian Wienert, Shiamaa Lazem, Jochen Meyer, Aneesha Singh, Andrew A. Bayor, Hajo Zeeb, and Johannes Schöning. 2020. Learning from mHealth Success Stories: Effective Interventions for Marginalised Populations. In Proceedings of the 22nd International Conference on Human-Computer Interaction with Mobile Devices and Services (Virtual) (MobileHCI '20). ACM, New York, NY, USA. https://doi.org/10.1145/3406324.3424653

[15] Catherine D'Ignazio and Lauren F Klein. 2020. Data feminism. MIT Press.

[16] Tanja Ertl, Konstantin Aal, Hoda Diraoui, Peter Tolmie, and Volker Wulf. 2020 Psychosocial ICT: The Potential, Challenges and Benefits of Self-help Tools for Refugees with Negative Mental Stress. In Proceedings of 18th European Conference on Computer-Supported Cooperative Work. European Society for Socially Embedded Technologies (EUSSET).

[17] Tanja Ertl, Sebastian Taugerbeck, Margarita Esau, Konstantin Aal, Peter Tolmie and Volker Wulf. 2019. The Social Mile-How (Psychosocial) ICT can Help to Promote Resocialization and to Overcome Prison. Proceedings of the ACM on Human-Computer Interaction 3, GROUP (2019), 1-31.

[18] Suzanne Fitzpatrick and Mark Stephens. 2014. Welfare regimes, social values and homelessness: Comparing responses to marginalised groups in six European countries. Housing Studies 29, 2 (2014), 215-234.

[19] Aakash Gautam, Chandani Shrestha, Andrew Kulak, Steve Harrison, and Deborah Tatar. 2018. Participatory tensions in working with a vulnerable population. In Proceedings of the 15th Participatory Design Conference: Short Papers, Situated Actions, Workshops and Tutorial-Volume 2.1-5.

[20] Danilo Giglitto, Shaimaa Lazem, and Anne Preston. 2018. In the eye of the student: an intangible cultural heritage experience, with a human-computer interaction twist. In Proceedings of the 2018 CHI Conference on Human Factors in Computing Systems. 1-12.

[21] Foad Hamidi, William Easley, Amy Hurst, Aaron Massey, and Helena Mentis. 2020 Position Paper: Facilitating Privacy Conversations with Vulnerable Populations. https://networkedprivacy2020.files.wordpress.com/2020/04/13_hamidi.pdf

[22] Richard Heeks. 2017. A structural model and manifesto for data justice for international development. Development Informatics Working paper 69 (2017).

[23] Lilly Irani, Janet Vertesi, Paul Dourish, Kavita Philip, and Rebecca E. Grinter. 2010. Postcolonial Computing: A Lens on Design and Development. In Proceedings of the SIGCHI Conference on Human Factors in Computing Systems (Atlanta, Georgia, USA) (CHI '10). Association for Computing Machinery, New York, NY, USA, 1311-1320. https://doi.org/10.1145/1753326.1753522

[24] Rikke Bjerg Jensen, Lizzie Coles-Kemp, and Reem Talhouk. 2020. When the Civic Turn Turns Digital: Designing Safe and Secure Refugee Resettlement. In Proceedings of the 2020 CHI Conference on Human Factors in Computing Systems (Honolulu, HI, USA) (CHI '20). Association for Computing Machinery, New York, NY, USA, 1-14. https://doi.org/10.1145/3313831.3376245

[25] Michael Katell, Meg Young, Dharma Dailey, Bernease Herman, Vivian Guetler Aaron Tam, Corinne Bintz, Daniella Raz, and PM Krafft. 2020. Toward situated interventions for algorithmic equity: lessons from the field. In Proceedings of the 2020 Conference on Fairness, Accountability, and Transparency. 45-55.

[26] Q. Vera Liao, Daniel Gruen, and Sarah Miller. 2020. Questioning the AI: Informing Design Practices for Explainable AI User Experiences. In Proceedings of the 2020 CHI Conference on Human Factors in Computing Systems (Honolulu, HI, USA) (CHI '20). Association for Computing Machinery, New York, NY, USA, 1-15. https://doi.org/10.1145/3313831.3376590

[27] Pallab Majumder, Michelle O’Reilly, Khalid Karim, and Panos Vostanis. 2015 'This doctor, I not trust him, I'm not safe': The perceptions of mental health and services by unaccompanied refugee adolescents. International journal of social psychiatry 61, 2 (2015), 129-136.

[28] Nicole Martinez-Martin, Thomas R Insel, Paul Dagum, Henry T Greely, and Mildred K Cho. 2018. Data mining for health: staking out the ethical territory of digital phenotyping. NPJ digital medicine 1, 1 (2018), 1-5.
[29] D. Harrison Mcknight, Michelle Carter, Jason Bennett Thatcher, and Paul F. Clay. 2011. Trust in a Specific Technology: An Investigation of Its Components and Measures. ACM Trans. Manage. Inf. Syst. 2, 2, Article 12 (July 2011), 25 pages. https://doi.org/10.1145/1985347.1985353

[30] Varun Mishra, Akane Sano, Saeed Abdullah, Jakob E. Bardram, Sandra Servia, Elizabeth L. Murnane, Tanzeem Choudhury, Mirco Musolesi, Giovanna Nunes Vilaza, Rajalakshmi Nandakumar, and Tauhidur Rahman. 2020. 5th International Workshop on Mental Health and Well-Being: Sensing and Intervention. In Adjunct Proceedings of the 2020 ACM International foint Conference on Pervasive and Ubiquitous Computing and Proceedings of the 2020 ACM International Symposium on Wearable Computers (Virtual Event, Mexico) (UbiComp-ISWC '20). Association for Computing Machinery, New York, NY, USA, 474-476. https://doi.org/10. $1145 / 3410530.3414615$

[31] Sachin R. Pendse, Naveena Karusala, Divya Siddarth, Pattie Gonsalves, Seema Mehrotra, John A. Naslund, Mamta Sood, Neha Kumar, and Amit Sharma. 2019. Mental Health in the Global South: Challenges and Opportunities in HCI for Development. In Proceedings of the 2nd ACM SIGCAS Conference on Computing and Sustainable Societies (Accra, Ghana) (COMPASS '19). Association for Computing Machinery, New York, NY, USA, 22-36. https://doi.org/10.1145/3314344.3332483

[32] Jay Rajasekera. 2020. How AI Can Bring About Social Good. Retrieved Nov 23, 2020 from https://development.asia/explainer/how-ai-can-bring-about-socialgood.

[33] Emrys Shoemaker, Gudrun Svava Kristinsdottir, Tanuj Ahuja, Dina Baslan, Bryan Pon, Paul Currion, Pius Gumisizira, and Nicola Dell. 2019. Identity at the margins: examining refugee experiences with digital identity systems in Lebanon, Jordan, and Uganda. In Proceedings of the 2nd ACM SIGCAS Conference on Computing and Sustainable Societies. 206-217.

[34] Aneesha Singh, Nikki Newhouse, Jo Gibbs, Ann E Blandford, Yunan Chen, Pam Briggs, Helena Mentis, Kate M Sellen, and Jakob E Bardram. 2017. HCI and health: Learning from interdisciplinary interactions. In Proceedings of the 2017 CHI Conference Extended Abstracts on Human Factors in Computing Systems. 1322-1325.

[35] Katta Spiel, Emeline Brulé, Christopher Frauenberger, Gilles Bailly, and Geraldine Fitzpatrick. 2018. Micro-Ethics for Participatory Design with Marginalised Children. In Proceedings of the 15th Participatory Design Conference: Full Papers - Volume 1 (Hasselt and Genk, Belgium) (PDC '18). Association for Computing Machinery, New York, NY, USA, Article 17, 12 pages. https://doi.org/10.1145/ 3210586.3210603

[36] Franziska Tachtler, Toni Michel, Petr Slovák, and Geraldine Fitzpatrick. 2020. Supporting the Supporters of Unaccompanied Migrant Youth: Designing for Social-Ecological Resilience. In Proceedings of the 2020 CHI Conference on Human Factors in Computing Systems (Honolulu, HI, USA) (CHI '20). Association for Computing Machinery, New York, NY, USA, 1-14. https://doi.org/10.1145/ 3313831.3376458

[37] Reem Talhouk, Konstantin Aal, Anne Weibert, Max Krüger, Volker Wulf, Karen Fisher, Franziska Tachtler, Suleman Shahid, Syed Ishtiaque Ahmed, and Ana Maria Bustamante Duarte. 2019. Refugees\&HCI SIG: Situating HCI Within Humanitarian Research. In Extended Abstracts of the 2019 CHI Conference on Human Factors in Computing Systems (Glasgow, Scotland Uk) (CHI EA '19). Association for Computing Machinery, New York, NY, USA, 1-4. https://doi.org/10.1145/3290607.3311754

[38] R. Talhouk, V. Vlachokyriakos, K. Aal, A. Weibert, S. Ahmed, K. Fisher, and V. Wulf. 2017. Refugees\&HCI Workshop: The Role of HCI in Responding to the Refugee Crisis. In Proceedings of the 8th International Conference on Communities and Technologies (Troyes, France) (C\&T'17). Association for Computing Machinery, New York, NY, USA, 312-314. https://doi.org/10.1145/3083671.3083719

[39] Reem Talhouk, Vasillis Vlachokyriakos, Anne Weibert, Konstantin Aal, Syed Ishtiaque Ahmed, Karen Fisher, and Volker Wulf. 2017. Refugees\&HCI Workshop: The Role of HCI in Responding to the Refugee Crisis. In Proceedings of the 2017 CHI Conference Extended Abstracts on Human Factors in Computing Systems (Denver, Colorado, USA) (CHI EA '17). Association for Computing Machinery, New York, NY, USA, 558-565. https://doi.org/10.1145/3027063.3027076

[40] Anja Thieme, Danielle Belgrave, and Gavin Doherty. 2020. Machine Learning in Mental Health: A Systematic Review of the HCI Literature to Support the Development of Effective and Implementable ML Systems. ACM Trans. Comput.Hum. Interact. 27, 5, Article 34 (Aug. 2020), 53 pages. https://doi.org/10.1145/ 3398069

[41] Shari Trewin, Sara Basson, Michael Muller, Stacy Branham, Jutta Treviranus, Daniel Gruen, Daniel Hebert, Natalia Lyckowski, and Erich Manser. 2019. Considerations for AI Fairness for People with Disabilities. AI Matters 5, 3 (Dec. 2019), 40-63. https://doi.org/10.1145/3362077.3362086

[42] Michael Ungar. 2013. Resilience after maltreatment: The importance of social services as facilitators of positive adaptation. Child Abuse \& Neglect 37, 2-3 (2013), $110-115$.

[43] Michael Ungar, Linda Liebenberg, Peter Dudding, Mary Armstrong, and Fons JR Van de Vijver. 2013. Patterns of service use, individual and contextual risk factors, and resilience among adolescents using multiple psychosocial services. Child abuse \& neglect 37, 2-3 (2013), 150-159. 
[44] Giovanna Nunes Vilaza, Raju Maharjan, David Coyle, and Jakob Bardram. 2020 Futures for Health Research Data Platforms From the Participants' Perspectives (NordiCHI '20). Association for Computing Machinery, New York, NY, USA Article 39, 14 pages. https://doi.org/10.1145/3419249.3420110

[45] John Vines, Róisín McNaney, Stephen Lindsay, Jayne Wallace, and John McCarthy. 2014. Special topic: Designing for and with vulnerable people. interactions 21, 1 (2014), 44-46.

[46] Ricardo Vinuesa, Hossein Azizpour, Iolanda Leite, Madeline Balaam, Virginia Dignum, Sami Domisch, Anna Felländer, Simone Daniela Langhans, Max Tegmark, and Francesco Fuso Nerini. 2020. The role of artificial intelligence in achieving the Sustainable Development Goals. Nature communications 11, 1 (2020), 1-10.

[47] Katharina Weitz, Dominik Schiller, Ruben Schlagowski, Tobias Huber, and Elisabeth André. 2019. "Do You Trust Me?": Increasing User-Trust by Integrating Virtual Agents in Explainable AI Interaction Design. In Proceedings of the 19th ACM International Conference on Intelligent Virtual Agents (Paris, France) (IVA '19). Association for Computing Machinery, New York, NY, USA, 7-9. https://doi.org/10.1145/3308532.3329441

[48] Heike Winschiers-Theophilus and Nicola J Bidwell. 2013. Toward an Afro-Centric indigenous HCI paradigm. International fournal of Human-Computer Interaction 29, 4 (2013), 243-255.

[49] Heike Winschiers-Theophilus, Shilumbe Chivuno-Kuria, Gereon Koch Kapuire, Nicola J Bidwell, and Edwin Blake. 2010. Being participated: a community approach. In Proceedings of the 11th Biennial Participatory Design Conference. $1-10$.

[50] Heike Winschiers-Theophilus, Tariq Zaman, and Alvin Yeo. 2015. Reducing" white elephant" ICT4D projects: a community-researcher engagement. In Proceedings of the 7th International Conference on Communities and Technologies. 99-107. 